\title{
Disaster and Urbanism
}

This special issue of the International Journal of Disaster Risk Science on Disaster and Urbanism shares our empirical research and conceptual contribution to the discussion of risk, disaster, and war and conflict from the perspective of urbanism or urban studies. By contributing to the emerging study of extreme urban conditions, the special issue engages urbanism with disaster risk science, crosses geopolitical boundaries, exhibits a variety of sociocultural traditions, and unfolds several transdisciplinary intellectual debates.

Highly complex and diffuse risks, multiscalar vulnerabilities, increasingly rapid urbanizations, and the absence of integration of intellectual contributions from all disciplines that deal with various types of risks and disasters characterize today's world. Disasters are more complex than traumatic natural events, as they usually refract various vulnerable facets of disaster-affected cities and territories that normal urban entities would rarely represent. Such extreme conditions have exposed and highlighted fundamental urban phenomena with extraordinary sociological meanings, ecological impacts, geopolitical significances, socioeconomic dynamics, and hence require interdisciplinary urban studies.

Despite the collection of six research articles that are all contributed by architects and urbanists, the special issue presents a wide variety of research themes. The cases range from territories of meteorological risk and problem-laden hydrological and industrial settings in Italy and France to Sarajevo, a war-torn city in Bosnia and Herzegovina, and earthquake-affected regions in Japan and China. Approaches involved in these studies include territorial management, urban planning, urban design, housing recovery, policy analysis, historic documentation, and economic analogue. Taken together, this collection of articles foregrounds a joint emphasis on disaster and urbanism as a common framework within which to address our ecological, geopolitical, and socioeconomic as well as spatial considerations.

Starting from the European debate on urban interventions for disaster vulnerable territories, the "extreme city" is coined in the two articles by Paola Viganò and Lorenzo Fabian to describe the extreme conditions of high meteorological and hydrological risks encountered in the Venice lagoon and Veneto plain areas in northern Italy where there exist "diffuse cities." These cases present climate change as a multidimensional catalyst to define new relations in and approaches to territorial development and disaster risk reduction. Also in Viganò's study the case of Bordeaux, France extends our ability to understand and address broader ecological and environmental issues in "bad places." The concluding argument is that urbanism and urban design should, and can, integrate disaster risk and vulnerability concerns. Armina Pilav's article examines the extreme crisis experienced in Sarajevo during the Bosnia War through the analysis of documentary archives and the author's lived experience. This study explores the impacts of a conflict on urban conditions by highlighting sociopolitical, economic, and spatial phenomena, and illustrates the various resilient strategies developed by residents of Sarajevo during the war.

The conceptual contribution by William Siembieda uses analogs from contemporary economic theory to model postdisaster recovery interactions over time and at multiple locations. Friction and uncertainty prevent efficient and effective completion of the transactions between internal and external sectors that are needed for recovery, by adding time and resource costs or slowing the recovery process. In this transdisciplinary discourse the innovative application of economic concepts benefits post-disaster studies by bringing external inspirations to the field. The two case studies on post-disaster urban recovery by Elizabeth Maly and Yashimitsu Shiozaki and Yan Guo look at a complex matrix of urban dynamics in the process of housing reconstruction, strategic recovery, and visionary urban planning and design for redevelopment. Maly and Shiozaki uses housing reconstruction policy as their storyline, extensively explain the reconstruction processes and their evolutionary developments after the Hanshin-Awaji and Chuetsu earthquakes, and discuss the implications of policy change for disaster recovery in Tohoku, Japan. Using a people-centered concept, their study provides critiques of earlier disaster recovery policies and argues for the need to empower residents to make decisions about their housing reconstruction and to generate policies creating housing options that support the ability of all residents to reconstruct their homes and lives. Yan Guo, in his study of the large-scale post-disaster reconstruction in Sichuan, China, provides alternative perspectives and design proposals for urban redevelopment based on an urbanistic interpretation of top-down Chinese urban planning and territorial management. At the end of the special issue, an international congress report from Keio University discusses the connections between disaster recovery and resilience building in the context of climate change.

"Urban resilience" or resilience in the urban context and in relation to disaster risks has been the key underlying concept in this collection. Through the experience of the extreme city and in the context of climate change risks, Fabian confronts paradigms of resilience with resistance. Viganò argues for urban resilience as an open possibility for future adjustments, through the provision of public space and flexible and adaptive composition in the urban fabric. Likewise, Guo seeks the 
possibility of enhancing urban resilience in the post-disaster Sichuan recovery through an alternative redevelopment scenario that focuses on sociospatial coherence and temporal continuity. Moreover, Pilav documents the pattern of urban resilience in a war-torn city's context, and provides evidence for how the urban infrastructure, civic defense and evacuation practices, and even the supply organizations of a city can become more resilient through the course of a war.

This special issue is a collection of knowledge based on a diverse set of contemporary research on disasters and urbanism that was initiated from nothing but shared enthusiasm for the topic, that was constructed on day-to-day collaboration and exchange among many individuals, and that was completed with great determination and patience. Therefore, the guest editors and the journal editorial team extend their sincere appreciation to all contributors and reviewers for their admirable effort and insightful knowledge, support, and assistance.

\author{
Guest Editors \\ Yan Guo \\ Paola Viganò \\ Bernardo Secchi \\ Peijun Shi \\ Editor-in-Chief
}

Open Access This article is distributed under the terms of the Creative Commons Attribution License which permits any use, distribution, and reproduction in any medium, provided the original author(s) and source are credited. 